\title{
A Novel Flow Control Valve for POCT Chip based on Micro Rectangular Groove Array
}

\author{
Yang Xie ${ }^{1,2, a)}$, Zhiyuan $\mathrm{Gao}^{3, b)}$, Weiqing Cheng ${ }^{3, \mathrm{c})}$, Ming $\mathrm{Li}^{5, \mathrm{~d}}$, Wei \\ $\operatorname{Han}^{1,4, \mathrm{e})}$ and Hui You ${ }^{1,2, \mathrm{f}, *)}$ \\ ${ }^{1}$ Institute of Intelligent Machines, Chinese Academy of Sciences, Hefei 230031, China \\ ${ }^{2}$ University of Science and Technology of China, Hefei 230026, China \\ ${ }^{3}$ Hefei university of technology, Hefei 230011, China \\ ${ }^{4}$ Center of Medical Physics and Technology, Chinese Academy of Sciences, Hefei 230031, China \\ ${ }^{5}$ The First Affiliated Hospital of USTC, AnHui Provincial Hospital \\ a)yxie9@mail.ustc.edu.cn \\ b)15212920245@163.com \\ c)chengweiqing@mail.hfut.edu.cn \\ d)liming19831002@163.com \\ e) hanw@hfcas.ac.cn \\ ${ }^{\mathrm{f}}$ hyou@iim.ac.cnh
}

\begin{abstract}
A novel flow control valve for point-of-care testing (POCT) chip, a micro rectangular groove array structure with capillary force as driving force, was proposed in this paper for the purposes of flowing controllable, spontaneous filling, low cost, and biological compatibility. The chip comprises two PMMA-based plates made of hot-embossing. The base plate and cover plate are connected by hot-bonding after ultrasonic cleaning and plasma treatment. An experiment has been carried out with deionized water to verify the control performance of fluid flow time in POCT chip by this method. The result convincingly indicates that this novel flow control valve could realize the function of controlling fluid flow, which meets the required time need for biochemical reactions. Compared with the controlling time of other previous methods in the POCT chip, the design in this study could make it to approximately 193 205 seconds. Therefore, this work has a great potential and broad prospects of the application in POCT chip, such as the portable devices in the medical testing field.
\end{abstract}

Keywords: Flow Control Valve, POCT Chip, Micro Rectangular Groove Array

\section{INTRODUCTION}

Point-of-care testing(POCT), a laboratory-medicine discipline, is evolving rapidly into the analytical scope and 
clinical application ${ }^{[1]}$. POCT aims at achieving fast and convenient testing by portable detecting instruments, thus making it possible to reduce costs as well as the waiting time and change the present situation that medical examination put more dependence on high-end equipment and testing professionals ${ }^{[2]}$. This modern variety of laboratory medicine is characterized by minimizing instrument size and the increasing use of current information technology ${ }^{[3]}$. More and more occasions used POCT due to the fact that these analytical devices can process a whole blood sample in a simple manner, allowing untrained staff to carry out laboratory diagnostics, shortening the time ofsample acquisition and analysis ${ }^{[4]}$. As a result of their low cost and good operability, POCT has been especially important to improve the integrity of the current medical system and promote a wider range of medical popularization, especially for those with limited mobility and poor medical conditions ${ }^{[5]}$.

The flow control of the microfluidic is a key to the study of the fluid microelectronic mechanical system(MEMS) and many of them can be attributed to scale effect and surface effect ${ }^{[6]}$. The fluid flow in a micro channel can be, concrete realized by two approaches: surface modification an microstructure on the surface of the channel. Surface modification refers to give the surface new properties under the precondition of maintaining the original properties of materials or products, such as hydrophilic and biocompatibility. Commonly used methods include ultrasonic surface treatment, surface coating treatment, irradiation surface modification technology. Jingmin $\mathrm{Li}$ used surface modification to control fluid front motion in microfluidic devices ${ }^{[7]}$. Surface microstructure refers to the change of surface structure under the premise of ensuring basic functions. Common methods include using the variation principle of the variable cross-section and change the fluid infiltration model. Xiaoyu Ding uses it to control fluid flow ${ }^{[8]}$. For POCT chip, the most important one is the flow control that based on microfluidic control. Due to its biological compatibility, the method of changing the surface structure of micro channel has been used in POCT chips. Of which, the micro cylinder array is most widely studied, which is normally manufactured by complicated and costly MEMS technology, e.g. lithography. Cong Yu proposed a flow control valve based on thermally responsive monolithic polymers, which also required a complicated manufacturing process ${ }^{[9]}$. Therefore, the development of the surface microstructures with good hydrophilic or hydrophobic property and low manufacturing cost is required, which is suitable for the application of flow speed control in the POCT chips.

In this paper, a novel and practical approach to the microstructure-based time-lapse self-opening valve will be demonstrated.

\section{PRINCIPLE}

\section{Capillary phenomenon}

The capillary phenomenon is one of the most common physical phenomena in nature. From 1709, Francis Hawksbee, a British scientist, observed the capillary phenomenon for the first time until Hans-Jurgen Butt introduced it in 2009 to quantitatively describe the size of the capillary force by using continuous medium theory ${ }^{[10]}$. After centuries of exploration, humans have uncovered the mystery of the capillary phenomenon. The spontaneous of the capillarity make it possible to drive microfluidic device without external powers. Meanwhile, it also verified the advantages of portability and low cost of the POCT device that based on the capillary phenomenon.

\section{Surface wetting phenomena}

Surface wetting phenomenon refers to the process that a liquid on a solid surface replaces another incompatible 
liquid $^{[11]}$. The infiltration or not depends on the size of the intermolecular force between the solid-liquid phase, which can be explained by the physical models shown in FIGURE 1.

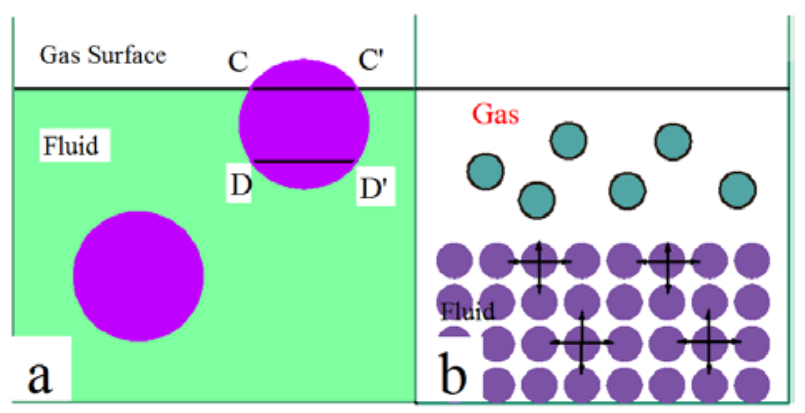

FIGURE 1. (a) A physical model of two molecular between the solid-liquid phases to explain surface wetting phenomenon. (b) Describe the molecular configuration of gas-liquid and the intermolecular force between the solid-liquid phase.

As is shown in FIGURE 1(a), A and B are the same, CC' and DD' are symmetrical about the origin of B. It is assumed that when the solid-liquid action is applied, the intermolecular force of solid phase is greater than that of the liquid phase, and the solid phase is not uniformly contracted due to stress, just as shown in FIGURE 1(b). The liquid phase molecular are close to the solid phase, and the infiltration phenomenon occurs. On the contrary, if the solid-liquid interaction is less than the intermolecular force of the liquid phase, the infiltration will not occur. Meanwhile, a small drop deposited on a solid that meets the solid surface with a well-defined angle. This angle and the shape of the drop are due to the existence of surface tension associated with each of the interfaces present in this system: liquid/gas, solid/liquid, and solid/gas interfaces coexist. This system tends to minimize the total surface energy, and in this way determines the value of the contact angles ${ }^{[12]}$. So the infiltration can be explained by the surface tension of material: When the surface tension of the material is greater than the surface tension of water, the material is hydrophilic, and the greater the surface tension, the stronger the hydrophilic. When the surface tension of the material is less than the surface tension of water, the material is hydrophobic, the surface tension is smaller, the hydrophobic is stronger, and the hydrophobicity can be described by contact Angle ${ }^{[13]}$. In a certain range, the more hydrophobic, the greater the contact angle. Therefore, the indirect contact between surface wetting phenomenon and contact angles can be established. FIGURE 2 shows the contact angle model.

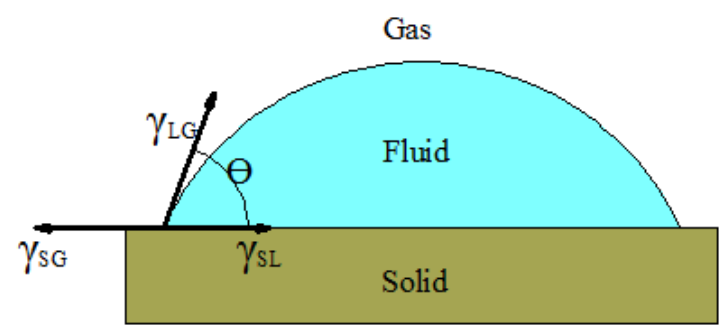

FIGURE 2. Solid-liquid-gas three phase contact angel

Ideally, contact angle and the solid-liquid-gas can be described by Young’s equation:

$$
\cos \theta=\left(\gamma_{S G}-\gamma_{S L}\right) / \gamma_{L G}
$$


Here, $\gamma_{S G}, \gamma_{S L}$, and $\gamma_{L G}$ are, respectively, the solid-vapor, solid-liquid, and liquid-vapor interfacial tension and $\theta$ is the contact angle ${ }^{[14]}$.

\section{Contact angle model of microstructure surface}

On the actual surface, due to the existence of microstructure, the surface roughness of the channel will be changed, which will lead to the change of contact angle between the solid-liquid phase. Therefore, there is a certain deviation from the apparent contact angle and the theoretical contact angle ${ }^{[15]}$.

In response to this phenomenon, the scientist Wenzel proposed the Wenzel model under the precondition of the rules and grooves on which the liquid droplets completely filled the solid surface ${ }^{[16]}$, as shown in FIGURE 3(a). It considered that the liquid is completely saturated and there is no gas phase in the solid-liquid contact interface. The conclusion is that the presence of roughness makes the hydrophilic material more hydrophilic and hydrophobic material more hydrophobic.
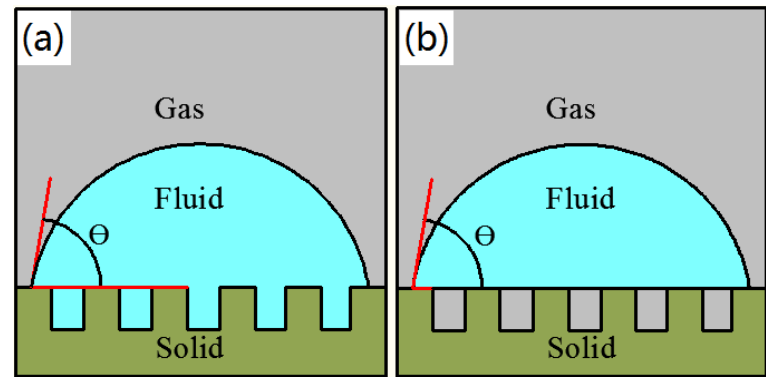

FIGURE 3. (a)Wenzel model of droplet on solid surface. (b) Cassie model of droplet on solid surface.

On the basis of the Wenzel model, scientists Cassie and Baxter proposed the Cassie model under the premise that the droplet did not fully fill the irregular grooves of the solid surface ${ }^{[17]}$, as shown in FIGURE 3(b).It considered that the liquid is not completely saturated in solid-liquid contact interface, and there is the gas phase. The conclusion is that the presence of roughness makes both hydrophilic and hydrophobic materials more hydrophobic.

\section{Influence of microstructure on surface wettability}

In order to study the relationship between microstructure and surface wettability, based on Wenzel and Cassie model, the microstructure model of surface rules was studied ${ }^{[18]}$. In the thermodynamic equilibrium state, Wenzel derived an equation similar to Young's equation ${ }^{[19]}$ :

$$
\cos \theta=\frac{r\left(\gamma_{S G}-\gamma_{S L}\right)}{\gamma_{L G}}
$$

In which, $\mathrm{r}$ is the surface roughness factor, the calculation formula for $\mathrm{r}$ is

$$
\mathrm{r}=\frac{s_{a}}{s_{p}}
$$

Among them, $S_{a}$ is the actual contact area and $S_{p}$ is the apparent contact area. For the micro rectangular array model, the Wenzel model is used, as shown in FIGURE 


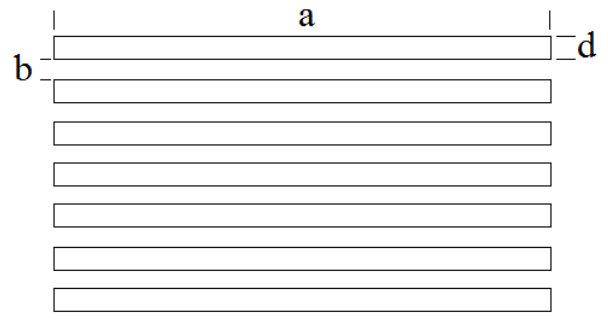

FIGURE 4. Enlarge figure of the regular micro rectangular groove array model

$$
\mathrm{r}=1+\frac{\pi \mathrm{dh}}{2 \mathrm{ab}}
$$

It is easy to get that the wettability of micro rectangular groove array is related to dh/ab. So change the size of micro rectangular groove array, influence surface wettability, and control microfluidic flow.

\section{METHOD}

\section{Design and fabrication of time valve}

Considering the characteristics of POCT chip, we choose PMMA, a kind of polymer, as the basic material due essentially to their low cost, easy processing and good biological compatibility ${ }^{[20]}$. FIGURE 5(a) shows the design of time valve.

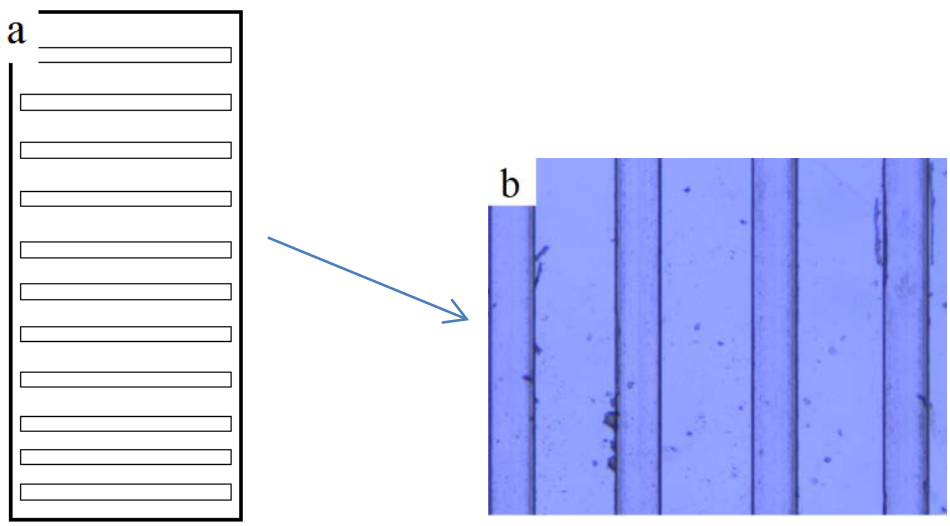

FIGURE 5. (a) time valve, consisting of a series of micro rectangular grooves. (b) Micro rectangular groove array’s structure of part B under the microscope.

In order to meet enough reaction time between samples and markers. The timing valve plays an important role in POCT chip. It is used to control fluid velocity and it is made up of a series of micro rectangular grooves. Its length is 5mm, and fluid flow through it at a controlled pace. FIGURE 5(b) shows the micro rectangular groove array's structure of part B under the microscope.

The base plate with a micro channel 2mm wide, 100um deep, and 4cm long shown schematically in Figure. 5(a) was used in this study. Briefly, PMMA was preprocessed on CNC milling machine to get a uniform surface and the 
suitable size ( $35 \mathrm{~cm}$ wide, $80 \mathrm{~cm}$ length and $2 \mathrm{~mm}$ thick) of stock. Next, we need a male mold with the complementary shape of the channel on a piece of metal. The stock is then thermo-pressed at $105^{\circ} \mathrm{C}$ for 90 mins, after been cool, using an ultrasonic cleaning machine for 10 minutes. Then use plasma cleaner to process PMMA in a vacuum for 2 minutes once it's gray. Use the same method to get the cover plate with an injection port shown in FIGURE 5(c). Finally, connect the base plate and the cover plate by hot-bonding at $75^{\circ} \mathrm{C}$ for 20 minutes, using isopropanol, which the volume fraction is $75 \%$, as an auxiliary solution.

\section{Experimental}

For purpose of verifying the control function of the microstructure, the micro rectangular groove array of the same structure is designed, which consists of 12 micro rectangular grooves. The depth of micro rectangular is 80um and the width is $3.4 \mathrm{~mm}$, and the length is $20 \mathrm{um}$. The experiment was performed on the same batch of 18 chips, where the liquid is deionized water and the flow time of the deionized water in the straight pipe is acquired and the length of the straight pipe iss six times the length of the time valve part.

\section{Results and discussions}

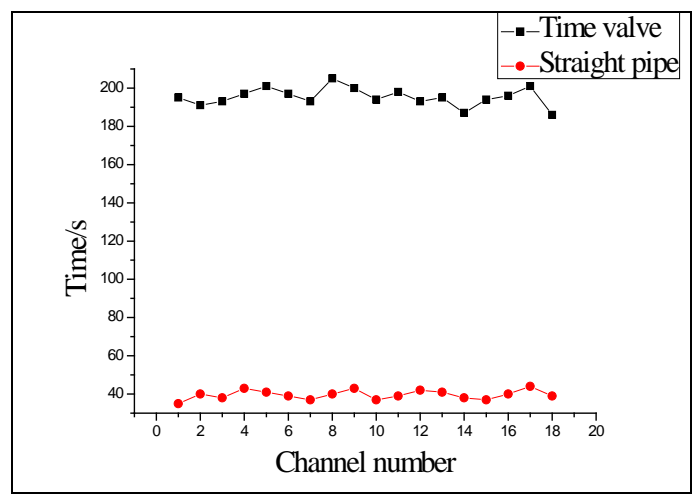

FIGURE 6. The flow time of the 18 experiments in the time valve part

As shown in FIGURE 6, we compare the time that fluid flows through the time valve part and the straight pipe. Obviously, the time that fluid flow through time valve part is longer than the time that fluid flow through the straight pipe, which demonstrates the control effect of micro rectangular groove array.

TABLE 1. The analysis of the data

\begin{tabular}{lcc}
\hline & Time valve & Straight pipe \\
\hline Average(s) & 195 & 39.6 \\
RSD(\%) & 4.78 & 2.43 \\
\hline
\end{tabular}

Comprehensive analysis table 1 is available, the flow of deionized water is relatively stable when passing through the time valve part and straight pipe. It's average time is 195s, the standard deviation are 4.78, and the flow time of fluid fluctuating within a certain range. The average time of the unprocessed straight pipe is $39.6 \mathrm{~s}$ and the standard deviation is 2.43. Compare the difference between time valve part and straight pipe. It is easy to obtain that the micro rectangular groove array can control the fluid flow, but it also raises the question about instability. The 
analysis may be due to the failure of the surface or microstructure of the straight pipe during machining or bonding.

\section{CONCLUSIONS}

Based on the POCT, a critical flow control valve with the capillary force as the driving force has been proposed in this study, which could satisfy the needs of flowing controllable, spontaneous filling, low cost, and biological compatibility. Then the flow control mechanism of the microstructure is explained and the empirical formula of the micro rectangular groove array structure is given to the Wenzel model. Meanwhile, many experiments of the same micro rectangular groove array structure have been carried out to verify the design's validity and stability. The experimental results show that the micro rectangular groove array structure could meet the design requirements of the POCT chip and effectively control the fluid flow.

\section{ACKNOWLEDGMEN}

This work was financially supported by the Key Research and Development Plan Project(1704a0802157) of Anhui , the Science and Technology Plan Project of Anhui (1501031088). The communication author is Hui You.

\section{REFERENCES}

1. Luppa P B, Müller C, Schlichtiger A, et al. Point-of-care testing (POCT): Current techniques and future perspectives[J]. Trends in Analytical Chemistry, 2011, 30(6):887-898.

2. Tonello S, Serpelloni M, Lopomo N F, et al. Preliminary study of a low-cost point-of-care testing system using screen-printed biosensors: For early biomarkers detection related to Alzheimer Disease[C]// IEEE International Symposium on Medical Measurements and Applications. IEEE, 2016:238-246.

3. Arai Y, Iinuma T, Takayama M, et al. The Tokyo Oldest Old Survey on Total Health (TOOTH): A longitudinal cohort study of multidimensional components of health and well-being[J]. Bmc Geriatrics, 2010, 10(1):35.

4. TANG Y H,GONG CH X, ZHOU Y M.New field for medical test-POCT[J].Medical Innoration of China,2012,9(23):153-154.

5. Anderson D A, Crowe S M, Garcia M. Point-of-Care Testing[J]. Current Hiv/aids Reports, 2011, 8(1):31-37.

6. Lewis D M, Gerecht S. Microfluidics and biomaterials to study angiogenesis[J]. Current Opinion in Chemical Engineering, 2016, 11:114-122.

7. Li J, Liang C, Wang S, et al. Using serrated edges to control fluid front motion in microfluidic devices[J]. Microsystem Technologies, 2017:1-8.

8. Ding X, Harris T A L. Review on penetration and transport phenomena in porous media during slot die coating[J]. Journal of Polymer Science Part B Polymer Physics, 2017, 55(22).

9. Yu $†$ C, Senol Mutlu $\ddagger$, Ponnambalam Selvaganapathy $\ddagger$, et al. Flow Control Valves for Analytical Microfluidic Chips without Mechanical Parts Based on Thermally Responsive Monolithic Polymers[J]. Analytical Chemistry, 2003, 75(8):1958-1961.

10. Suh K Y, Kim Y S, Lee H H. Capillary Force Lithography[J]. Advanced Materials, 2001, 13(18):1386.

11. Starov V M, Velarde M G. Surface forces and wetting phenomena.[J]. J Phys Condens Matter, 2009, 21(46):3543-3549.

12. QuÉ D, ré. Surface wetting: Model droplets[J]. Nature Material, 2004, 3(2):79-80.

13. Shi D, Li D, Gao G. Relation between surface tension and microstructural modification in Al-Si alloys[J]. 
Materials Characterization, 2008, 59(11):1541-1545.

14. Seveno D, Blake T D, De C J. Young's equation at the nanoscale.[J]. Physical Review Letters, 2013, 111(9):096101.

15. Masashi Miwa †, Akira Nakajima †, Akira Fujishima $\ddagger$, et al. Effects of the Surface Roughness on Sliding Angles of Water Droplets on Superhydrophobic Surfaces[J]. Langmuir, 2000, 16(13):5754-5760.

16. Wenzel R N. RESISTANCE OF SOLID SURFACES TO WETTING BY WATER[J]. Ind.eng.chem, 1936, 28(8):988-994.

17. A. B. D. Cassie, S. Baxter. Wettability of porous surfaces[J]. Trans Faraday Soc, 1944,40(1):546-551.

18. Yuan Q, Zhao Y P. Multiscale dynamic wetting of a droplet on a lyophilic pillar-arrayed surface[J]. Journal of Fluid Mechanics, 2013, 716(2):171-188.

19. Nosonovsky M. On the Range of Applicability of the Wenzel and Cassie Equations[J]. Langmuir, 2007, 23(19):9919-9920.

20. Shui M. Polymer surface modification and characterization of particulate calcium carbonate fillers[J]. Applied Surface Science, 2003, 220(1-4):359-366. 